Apidologie, 1970, 1 (4), 423-438.

\title{
LES EFFETS DU MODE D'ALIMENTATION ET DE LA GELÉE ROYALE SUR LA SURVIE DES ABEILLES D'ÉTÉ EN FONCTION DE L'ISOLEMENT
}

\author{
Über die Wirkung der Ernährungsart und des Königinnenfutter- \\ saftes auf die Lebensdauer isoliert gehaltener Sommerbienen
}

Guy SITBON

Laboratoire de Psychophysiologie, Faculté des Sciences, 67 - Strasbourg

\section{SUMMARY}

THE EFFECTS OF FEEDING METHOD AND " ROYAL JELLY 》

ON SUMMER BEES IN TERMS OF ISOLATION

$1^{\circ}$ Confined, isolated or grouped summer bees do not consume fresh " royal jelly $\triangleright$ and are sensitive to the liquid or solid aspeet of food.

$2^{\circ}$ The " royal jelly" mixed with honey and the lipid extracts of " royal jelly " mixed with candy have no favourable effect on the survival of summer bees grouped by $30 \mathrm{~s}$, and a negative effect on the survival of isolated summer bees.

\section{RESUMÉ}

On a utilisé dans les expériences du couvain d'été naissant. Différents types d'alimentation ont été administrés : miel pur liquide, candi, gelée royale pure ou extraits de gelée royale. Toutes les abeilles disposaient de pollen réduit en poudre. Si l'on présente aux abeilles 3 godets contenant respectivement du candi, du pollen et de la gelée royale, on constate que les insectes ont une répulsion pour cette dernière. La gelée royale est donc mélangée dans les expériences au miel et au candi.

La mortalité des abeilles groupées disposant de miel pur est très élevée. La cause principałe en est une rétention d'eau importante. Chez les abeilles isolées, les inconvénients de l'alimentation 
liquide se manifestent plus tardivement. La mortalité des abeilles groupées nourries d'un mélange de miel et $10 \%$ de getée royale est également très forte et due sans doute aussi à la rétention d'eau. Il n'y a pas de différence de mortalité statistiquement significative entre abeilles isolées et groupées. La gelée royale semble avoir une action négative sur la survie des abeilles isolées.

Quand les abeilles disposent de candi, il y a un effet de groupe très important sur la survie. Au contraire, si les abeilles sont nourries de miel, la différence de mortalité n'est pas significative. Les abeilles claustrées sont donc sensibles à la nature physique des aliments.

Dans d'autres expériences, on a utilisé des extraits de gelée royale mélangés à du candi. On constate que les extraits de gelée royale sont sans effet sur la survie des abeilles groupées. Cependant, ils ont une action négative sur la survie des abeilles d'été isolées. Autrement dit, un régime alimentaire à base d'extraits de gelée royale diminue la survie des abeilles d'été isolées.

\section{INTRODUCTION}

Après avoir montré que la différence de mortalité, notée en toutes saisons (Sitbon, $1967 c$ ) entre abeilles isolées et groupées nourries de candi, n'était due ni à une différence d'alimentation (Sitbon, 1967 b) ni à une perturbation du métabolisme des sucres, de l'eau et de l'azote (Sitbon, $1968 a, b$ ), j'ai cherché à connaître l'action des substances normalement présentes dans la ruche, et en particulier les effets de la gelée royale, sur la survie des abeilles d'été. C'est cette étude que je me propose d'exposer dans ce mémoire.

\section{MATÉRIEL ET MÉTHODES}

Dans toutes mes expériences, j'ai utilisé du couvain d'été naissant placé dans des étuves réglées à $32^{\circ} \mathrm{C} \pm 1{ }^{\circ} \mathrm{C}$ et maintenu à l'obscurité. Les cagettes d'isolement et les conditions de vie des abeilles claustrées sont identiques à celles décrites dans les expériences citées ci-dessus. Sauf indication contraire, chaque expérience a porté sur 176 abeilles isolées et 240 abeilles groupées par 30 , réparties en deux lots égaux. Le premier reçoit une alimentation additionnée de la substance à tester. Le second reçoit la même alimentation sans substance; il s'agit donc d'un lot témoin.

Toutes les abeilles ont disposé de pollen conservé à $-30^{\circ} \mathrm{C}$ et préalablement réduit à l'état de poudre fine.

La source d'hydrates de carbone est, tantôt un aliment liquide (miel du Gâtinais), tantôt un aliment solide, candi réalisé à froid, par mes soins, selon la recette suivante : $40 \mathrm{~g}$ de miel du Gâtinais $+115 \mathrm{~g}$ de sucre glace, soit un candi composé de $35 \%$ de miel par rapport au sucre glace.

$\mathrm{La}$ gelée royale à tester a été conservée au froid, à $-30^{\circ} \mathrm{C}$. Dans certaines expériences, je l'ai utilisée pure et dans d'autres j'ai eu recours à des extraits réalisés à l'éther. Ces extraits ont été obtenus de la façon suivante : la quantité de gelée royale nécessaire à l'expérience est pesée dans une ampoule à décanter, puis diluée dans une petite quantité d'eau. A la solution obtenue, j'ajoute un excès d'éther. Après une agitation vigoureuse et répétée, je laisse décanter le tout et obtiens alors deux phases : une phase aqueuse surmontée d'une phase éthérée. Je laisse s'écouler la première et récupère la seconde dans un mortier à large ouverture permettant une évaporation rapide de l'éther, accélérée par l'action d'un ventilateur.

La phase aqueuse est retraitée de la même manière 2 à 3 fois de suite, puis jetée. Les phases éthérées successives sont toutes recueillies. Après évaporation totale de l'éther, il reste dans le mortier un dépôt gras, jaunâtre, à odeur forte et acide. Cet extrait est repris par la quantité de miel devant intervenir dans la composition du candi $(40 \mathrm{~g})$, puis mélangé au sucre glace ( $115 \mathrm{~g})$. Ce candi ne diffère donc du candi témoin que par la présence des extraits de gelée royale obtenus après action de l'éther.

La mortalité a été contrôlée tous les jours.

Dans les calculs statistiques j'ai utilisé le test du $\chi^{2}$. 


\section{RÉSULTATS}

Au cours d'une expérience préliminaire, j'ai fourni aux abeilles claustrées, tant isolées que groupées, 3 godets contenant respectivement du candi, du pollen et de la gelée royale. L'observation des insectes a montré qu'ils se posaient indifféremment sur les godets à candi ou à pollen, mais évitaient celui à gelée royale. Mieux encore, si par aventure l'un d'entre eux tombait accidentellement sur le dernier type de godet, loin d'y demeurer, il s'en éloignait aussitôt, comme repoussé. La gelée royale est répulsive pour les abeilles. Au bout de quelques heures, la gelée royale se dessèche sous l'action de la chaleur de l'étuve, sans avoir apparemment été touchée.

Cette constatation m'a incité à mélanger la gelée royale à la source alimentaire habituelle, en l'occurence aux hydrates de carbone.

\section{A. - Action de la gelée royale mélangée à du miel}

Deux expériences similaires ont été menées de front sur du couvain naissant prélevé le 16 mai 1968. Chaque expérience a porté sur 2 lots identiques constitués chacun de 40 abeilles isolées et 120 abeilles groupées par 30 . Le premier lot dispose de pollen et d'un mélange de miel $+10 \%$ de gelée royale, le deuxième de pollen et de miel pur.

Ces expériences ont été poursuivies durant 28 jours. Les résultats obtenus sont transcrits sur les courbes des figures 1 et 2 .

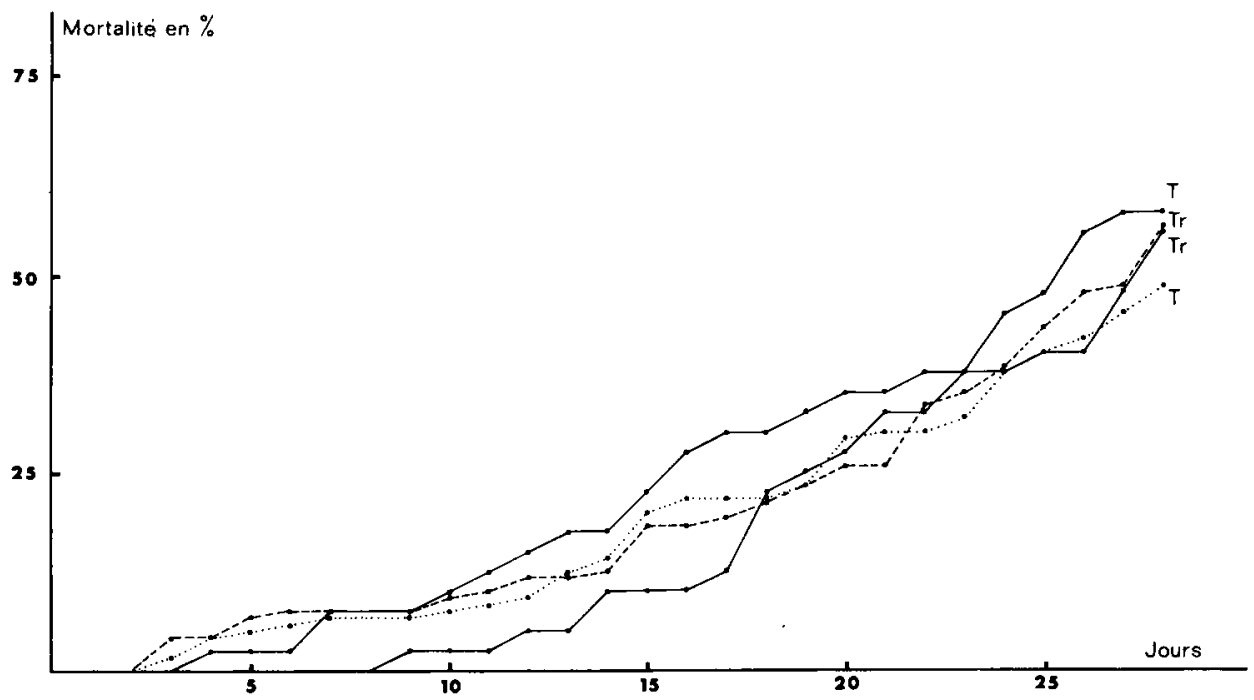

Fic. 1. - Action de la gelée royale mélangée à du miel

Tr : Abeilles traitées disposant de pollen et de miel $+10 \%$ de gelée royale.

T : Abeilles témoins disposant de pollen et de miel pur.

- : Abeilles isolées.

$--1 \ldots$ : Abeilles groupées.

Aвв. 1. - Wirkung von mit Honig vermischtem Königinnenfuttersaft

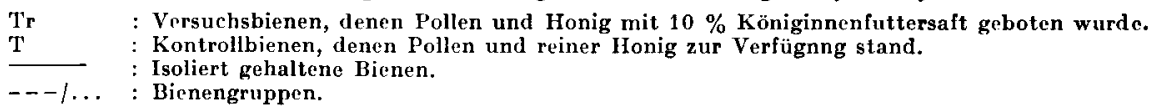




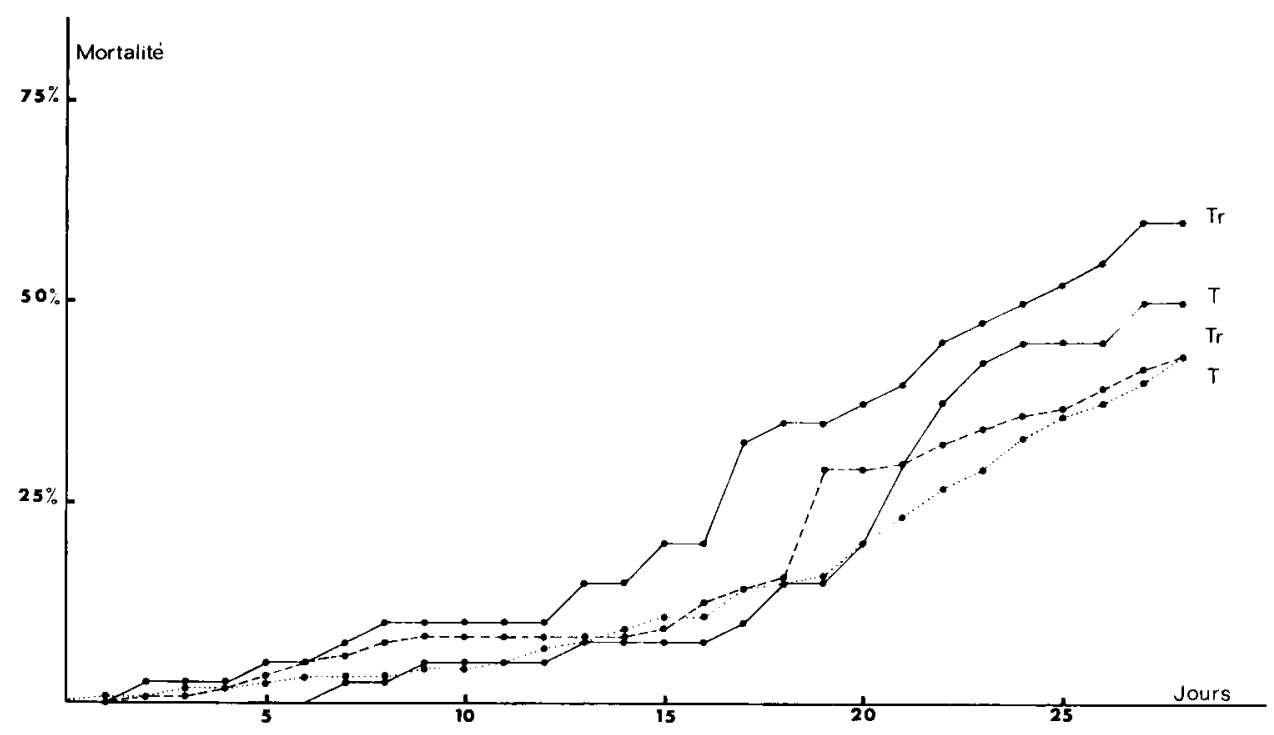

FIG. 2. - Action de la gelêe royale mélangée à du miel

Tr : Abeilles traitées disposant de pollen et de miel $+10 \%$ de gelée royale.

T : Abeilles témoins disposant de pollen et de miel pur.

: Abeilles isolées.

$--1 \ldots$ : Abeilles groupées.

Авв. 2. - Wirkung von mit Honig vermischtem Königinnenfuttersaft

Tr : Versuchsbienen, denen Pollen und Honig mit Zugabe von $10 \%$ Königinnenfutlersaft geboten wurde.

T : Kontrollbienen, die Pollen und reinen Honig erhielten.

: Isoliert gehaltene Bienen.

$--1 \ldots \quad$ : Bienengruppen.

Analyse et interprétation des courbes.

1. — Effets des aliments liquides.

a. Miel pur.

La constatation qui s'impose immédiatement est que la mortalité des abeilles groupées disposant de miel pur est anormalement élevée. Après 28 jours de claustration, la différence de mortalité entre abeilles groupées et abeilles isolées soumises au même régime alimentaire n'est plus statistiquement significative.

\begin{tabular}{|c|c|c|c|c|c|}
\hline \multicolumn{3}{|c|}{ Expérience 1} & \multicolumn{3}{|c|}{ Expérience 2} \\
\hline & mortes & vivantes & & mortes & vivantes \\
\hline Abeilles isolées . . . . . . . & 23 & 17 & Abeilles isolées . . . . . . & 20 & 20 \\
\hline Abeilles groupées...... & 58 & 62 & Abeilles groupées...... & 52 & 68 \\
\hline \multicolumn{3}{|c|}{$x^{2}=1,008$} & \multicolumn{3}{|c|}{$x^{2}=0,538$} \\
\hline
\end{tabular}


L'effet de groupe sur la survie a donc disparu et les abeilles groupées par 30 meurent aussi vite que les abeilles isolées. Ces résultats sont absolument opposés à ceux obtenus dans les expériences des années antérieures où les hydrates de carbone étaient distribués sous forme solide, à savoir de candi.

Cette mortalité exagérée des abeilles groupées est donc à rejeter sur la nature physique des aliments et plus précisément sur une rétention d'eau importante. On peut en effet constater que les insectes morts ont l'abdomen excessivement gonflé. Les abeilles vivant claustrées ne défèquent pas et de ce fait n'ont pas la possibilité d'éliminer l'excédent de liquide.

Chez les abeilles isolées, les inconvénients de l'alimentation liquide ne semblent se manifester que plus tardivement.

Cette hypothèse d'une rétention d'eau est confirmée par une expérience que j'exposerai plus loin.

\section{b. Miel $+10 \%$ de gelée royale.}

Comme précédemment, la mortalité des abeilles groupées, nourries cette fois d'un mélange de miel $+10 \%$ de gelée royale, est anormalement forte. Les effets de la rétention d'eau sont, là aussi, présents. Il n'y a pas de différence de mortalité statistiquement significative entre abeilles isolées et groupées soumises au même régime alimentaire.

\begin{tabular}{c|c|c|c|c|c}
\hline \multicolumn{2}{c|}{ Expérience 1 } & \multicolumn{2}{c}{ Expérience 2 } \\
\hline & mortes & vivantes & & mortes & vivantes \\
\hline Abeilles isolées........ & 22 & 18 & Abeilles isolées....... & 24 & 16 \\
\hline Abeilles groupées...... & 67 & 53 & Abeilles groupées...... & 52 & 68 \\
\hline$x^{2}=0,008$ & & $x^{2}=3,341$ \\
\hline
\end{tabular}

2. - Action de la gelée royale sur la survie.

Pour comprendre cette action, il convient donc de tenir compte des effets de la rétention d'eau.

a. Action chez les abeilles groupées.

Si l'on compare la survie des abeilles groupées nourries de miel pur à celle d'abeilles groupées disposant de miel $+10 \%$ de gelée royale, on constate qu'il n'y a pas de différence de mortalité statistiquement significative. 


\begin{tabular}{|c|c|c|c|c|c|}
\hline \multicolumn{3}{|c|}{ Expérience 1} & \multicolumn{3}{|c|}{ Expérience 2} \\
\hline & mortes & vivantes & & mortes & vivantes \\
\hline $\begin{array}{c}\text { Abeilles groupées trai- } \\
\text { tées } \ldots \ldots \ldots \ldots \ldots \ldots\end{array}$ & 67 & 53 & $\begin{array}{c}\text { Abeilles groupées trai- } \\
\text { tées . . . . . . . . } \ldots \ldots\end{array}$ & 52 & 68 \\
\hline $\begin{array}{c}\text { Abeilles groupées té- } \\
\text { moins } \ldots \ldots \ldots \ldots \ldots\end{array}$ & 58 & 62 & $\begin{array}{c}\text { Abeilles groupées té- } \\
\text { moins . . . . . . . . }\end{array}$ & 52 & 68 \\
\hline \multicolumn{3}{|c|}{$x^{2}=1,351$} & \multicolumn{3}{|c|}{$x^{2}=0,000$} \\
\hline
\end{tabular}

Autrement dit, la gelée royale semble sans effet sur la survie des abeilles groupées par 30, à moins que l'action de la rétention d'eau ne masque ces effets.

\section{b. Action chez les abeilles isolées.}

Il en est de même chez les abeilles isolées disposant de miel pur ou de miel additionné de $10 \%$ de gelée royale, quand on considére la fin de l'expérience, c'est-à-dire au bout de 28 jours d'isolement. Dans ce cas aussi, la différence de mortalité entre abeilles traitées et témoins n'est pas statistiquement significative.

\begin{tabular}{c|c|c|c|c|c}
\hline \hline \multicolumn{2}{c|}{ Expérience 1 } & \multicolumn{2}{c}{ Expérience 2 } \\
\hline mortes & vivantes & & mortes & vivantes \\
\hline Abeilles isolées traitées. & 22 & 18 & Abeilles isolées traitées. & 24 & 16 \\
\hline Abeilles isolées témoins. & 23 & 17 & Abeilles isolées témoins. & 20 & 20 \\
\hline
\end{tabular}

Comme précédemment, il semble que la gelée royale soit sans action sur la survie. Mais, comme je l'ai mentionné au début du paragraphe, il faut tenir compte des effets de la rétention d'eau, susceptibles de masquer l'influence de la gelée royale. Les expériences décrites montrent que chez les abeilles groupées disposant d'une alimentation liquide, la mortalité est anormalement élevée dès le début de l'expérience. Il est par conséquent difficile de déterminer ce qui est dû à la gelée royale $\epsilon t$ ce qui $\epsilon$ st dû à la rétention d'eau. Au contraire, pour les abeilles isolées soumises au même régime alimentaire, les courbes montrent que l'action des aliments liquides re se manifeste que tardivement. Autrement dit, il doit être possible d'étudier l'action de la gelée royale, si l'on se place avant le début des effets de la rétention d'eau et par conséquent de dissocier les deux actions.

En effet, si on compare les résultats obtenus après 17 jours d'isolement, on ccnstate que la mortalité des abeilles isolées nourries de miel $+10 \%$ de gelée royale est plus forte que celle des abeilles isolées disposant de miel pur. Cette 
différence est statistiquement significative au seuil de $5 \%$ pour l'expérience 1 et au seuil de $1 \%$ pour l'expérience 2 .

\begin{tabular}{|c|c|c|c|c|c|}
\hline \multicolumn{3}{|c|}{ Expérience 1} & \multicolumn{3}{|c|}{ Expérience 2} \\
\hline & mortes & vivantes & & mortes & vivantes \\
\hline Abeilles isolées traitées. & 12 & 28 & Abeilles isolées traitées, & 13 & 27 \\
\hline Abeilles isolées témoins. & 5 & 35 & Abeilles isolées témoins. & 4 & 36 \\
\hline \multicolumn{3}{|c|}{$x^{2}=3,660$} & \multicolumn{3}{|c|}{$x^{2}=6,050$} \\
\hline
\end{tabular}

Par conséquent, dans ces conditions expérimentales, la gelée royale semble avoir une action négative sur la survie des abeilles isolées, action masquée par la rétention d'eau si l'expérience se prolonge.

Il est évident que cette conclusion n'a de sens que si l'hypothèse formulée quant à l'action des aliments liquides est vraie, autrement dit, s'il y a réellement mortalité exagérée par suite de rétention d'eau. Pour le vérifier, j'ai réalisé une expérience permettant la comparaison de la survie d'abeilles isolées et groupées nourries de miel pur à celle d'abeilles isolées et groupées disposant de candi.

\section{B. - Comparaison des effets du miel pur et du candi}

L'expérience a porté sur du couvain naissant prélevé le 18 juin 1968. 88 abeilles isolées et 250 abeilles groupées par 50 ont reçu du candi et du pollen tandis que des lots identiques ont été nourris de miel pur. Les résultats obtenus sont consignés sur les courbes de la figure 3 .

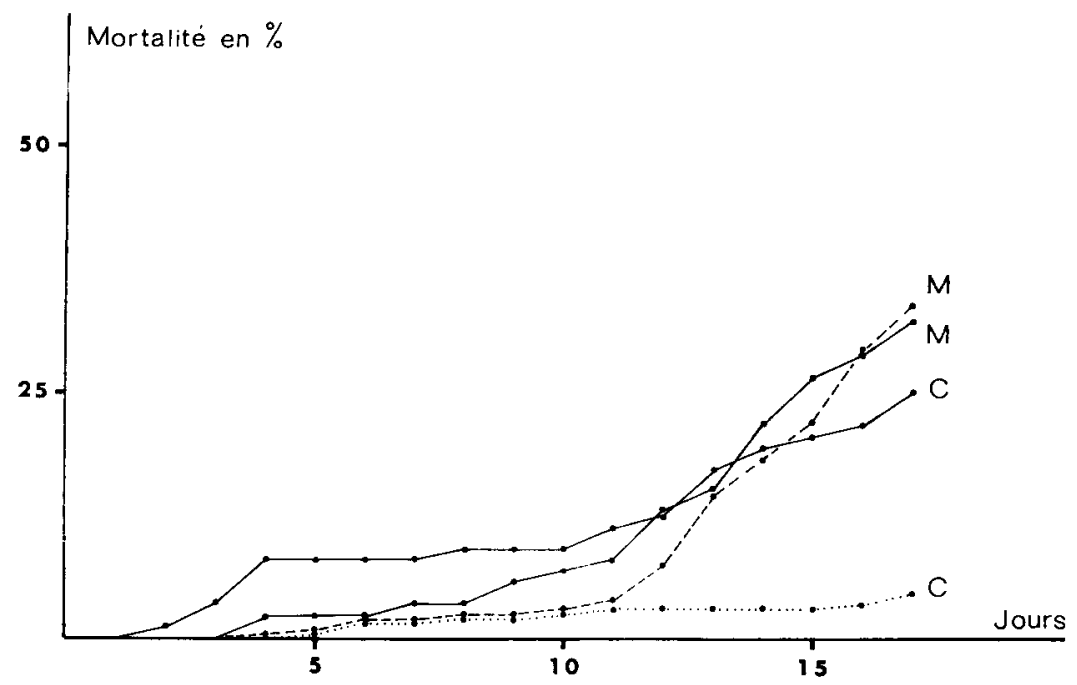

Fig. 3. - Influence du mode d'alimentation sur la mortalité

M : Abeilles nourries de pollen et de miel.

C : Abeilles nourries de pollen et de candi.

: Abeilles isolées.

$---1 \ldots:$ Abeilles groupées.

AвB. 3. - Einfluss der Ernährungsweise auf die Sterblichkeit

$\begin{array}{ll}\mathrm{M} & : \begin{array}{l}\text { mit Pollen und Honig ernährte Bienen. } \\ \mathrm{C}\end{array} \quad \begin{array}{l}\text { mit Pollen und Futterteig ernährte Bienen. } \\ \text { Isoliert gehaltene Bienen. }\end{array} \\ -,-\ldots & \text { : Bienengruppen. }\end{array}$ 
Analyse et interprétation des courbes.

Ces courbes permettent de vérifier que quand les abeilles disposent de candi il y a un effet de groupe très important, sur la survie. La différence de mortalité entre abeilles groupées et isolées est hautement significative à près de $1 \%$ oo.ooo.

\begin{tabular}{|c|c|c|c|}
\hline & mortes & vivantes & \\
\hline Abeilles isolées sur candi & 22 & 66 & $x^{2}=26,737$ \\
\hline $\begin{array}{c}\text { Abeilles groupées sur } \\
\text { candi. ............. }\end{array}$ & 9 & 191 & \\
\hline
\end{tabular}

Ceci confirme les résultats des expériences des années précédentes où les hydrates de carbone étaient donnés sous forme de candi.

$\mathrm{Au}$ contraire, si les abeilles sont nourries de miel, la différence de mortalité entre abeilles groupées et isolées n'est guère significative.

\begin{tabular}{l|c|c|c}
\hline & mortes & vivantes & \\
\cline { 2 - 3 } & 28 & 66 & \\
$\begin{array}{c}\text { Abeilles isolées sur miel. } \\
\begin{array}{r}\text { Abeilles groupées sur } \\
\text { miel............... }\end{array}\end{array}$ & 68 & 132 & $\varkappa^{2}=0,089$ \\
\hline \hline
\end{tabular}

L'effet de groupe disparait et les abeilles groupées meurent aussi vite que celles isolées, comme nous l'avons vu précédemment. Il est clair que cette différence entre les deux cas est imputable à la nature physique des aliments.

D'ailleurs, la mortalité des abeilles groupées nourries de miel est beaucoup plus importante que celle des abeilles groupées disposant de candi. Cette différence de mortalité est très hautement significative, à $1 \%$ oo.ooo.ooo.

\begin{tabular}{c|c|c|c}
\hline \hline & mortes & vivantes & \\
\hline $\begin{array}{r}\text { Abeilles groupées sur } \\
\text { miel................. }\end{array}$ & 68 & 132 & \\
\hline $\begin{array}{r}\text { Abeilles groupées sur } \\
\text { candi ............... }\end{array}$ & 9 & 191 & \\
\hline \hline
\end{tabular}

Chez les abeilles isolées cela est moins net. A la fin de l'expérience la différence de mortalité entre abeilles vivant sur candi et celles nourries de miel n'est pas significative.

\begin{tabular}{l|c|c|c}
\hline \hline & mortes & vivantes & \\
\hline Abeilles isolées sur miel. & 28 & 59 & $\chi^{2}=1,106$ \\
\hline Abeilles isolées sur candi & 22 & 66 & \\
\hline \hline
\end{tabular}


Cependant, on peut noter sur les courbes, à partir du $12^{\mathrm{e}}$ jour d'isolement, une inversion de la mortalité en faveur des abeilles vivant sur candi. Ce phénomène va en s'accentuant jusqu'au dernier jour d'expérience. Cette observation confirme l'idée d'une action tardive de l'alimentation liquide, chez les abeilles isolées. Mais elle souligne aussi le fait, qu'en début d'expérience, les abeilles isolées vivent mieux sur aliment liquide que sur aliment solide.

En résumé, cette expérience montre que les abeilles claustrées sont sensibles à la nature physique des aliments. Il semblerait que chez les abeilles groupées il y ait rétention d'eau importante dès le début, avec mortalité exagérée. Au contraire, les abeilles isolées semblent tirer profit d'une alimentation liquide pendant un certain temps, au-delà duquel les effets de la rétention d'eau commencent à se manifester et s'accentuent progressivement.

Il est par conséquent logique de penser que l'action de la gelée royale mélangée à du miel est plus ou moins masquée par cette rétention d'eau. Pour pallier cette difficulté et dissocier les 2 phénomènes, j'ai administré des extraits de gelée royale obtenus conformément à la technique décrite plus haut, et mélangés à du candi. Les animaux témoins ont été nourris de candi simple.

Dans ces expériences, les quantités de gelée royale traitée représentent successivement $20 \%, 10 \%$ et $5 \%$ du poids du candi simple utilisé dans le mélange.

Je parlerai de candi à $20 \%$, à $10 \%$ ou à $5 \%$, en sous-entendant qu'il s'agit de candi contenant les extraits provenant d'une quantité de gelée royale représentant cette proportion.

\section{C. - Action des extraits, à l'éther, de gelée royale}

Les conditions expérimentales sont celles décrites dans le chapitre matériel et méthodes. Chaque expérience a duré quinze jours et le couvain naissant a été prélevé respectivement le 25 avril, le 11 mai et le 30 mai 1969. Les résultats des trois expériences sont consignés sur les courbes des figures 4,5 et 6 .

Dans les trois expériences, on constate qu'il n'y a pas de différence de mortalité significative entre abeilles groupées nourries de candi témoin et abeilles groupées nourries de candi à 20,10 ou $5 \%$ de gelée royale.

\begin{tabular}{c|c|c|c|c|c}
\hline \hline \multicolumn{2}{c}{ Candi à $10 \%$} & \multicolumn{2}{c}{ Candi à $5 \%$} \\
\hline & mortes & vivantes & & & \\
\hline Abeilles traitées...... & 78 & 10 & mortes & vivantes \\
\hline Abeilles témoins....... & 62 & 26 & Abeilles traitées....... & 61 & 27 \\
\hline \multicolumn{2}{c|}{$x^{2}=8,939$} & & & 41 & 47 \\
\hline \hline
\end{tabular}




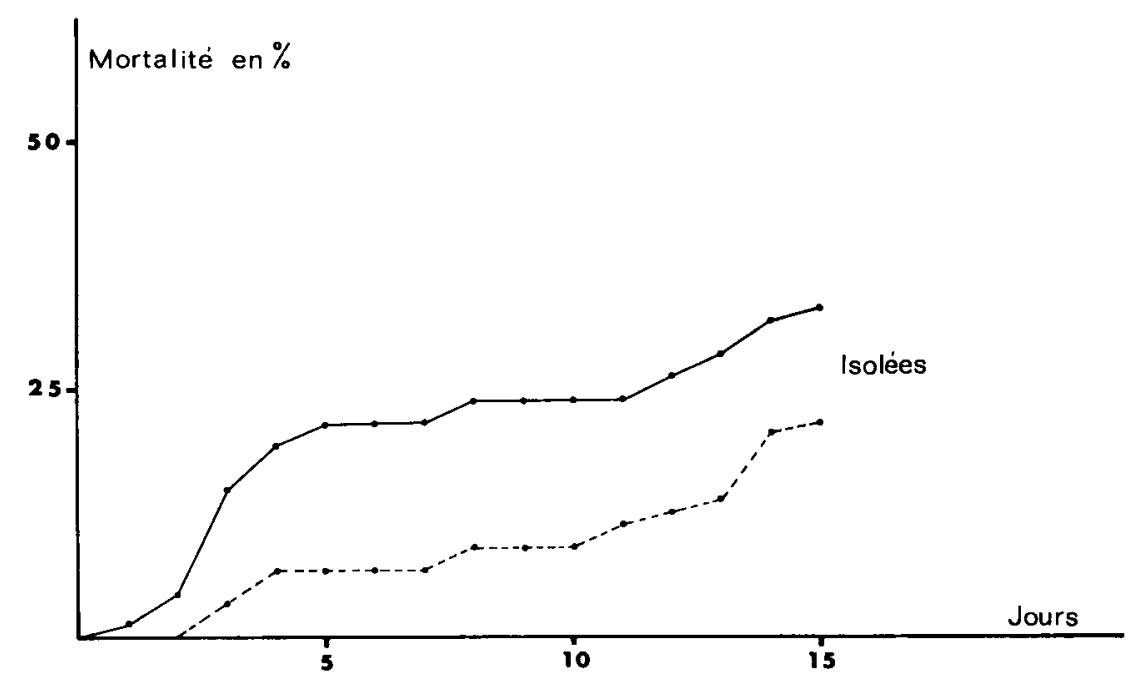

FIG. 4. - Action des extraits lipidiques de gelée royale

: Abeilles traitées disposant de pollen et de candi + extraits lipidiques provenant de $20 \%$ de gelée royale.

: Abeilles témoins disposant de pollen et de candi pur.

N. B. - Dans cette expérience, la mortalité des abeilles groupées est nulle pour les deux types d'alimentation.

Aвв. 4. - Wirkung der Königinnenfuttersafi-Extrakte

- $\quad$ : Versuchsbienen, mit Pollen, Futterteig und Extrakten aus $20 \%$ Königinnenfuttersaft ernährt.

- - : Kontrollbienen, mit Pollen und reinem Futterteig ernährt.

$N . B$. - In diesem Versuch ist die Sterblichkeit der Bienengruppen gleich null bei beiden Ernăhrungsarten.

Les extraits de gelée royale sont donc sans effet sur la survie des abeilles groupées tout comme nous l'avions déjà constaté à propos de la gelée royale entière, mélangée à du miel.

Il en va différemment pour les abeilles isolées. En effet, les abeilles isolées traitées meurent plus vite que les abeilles isolées témoins. Ainsi dans le cas des " candi à 10 et à $5 \%$ ", cette différence de mortalité est significative à $1 \%$. 


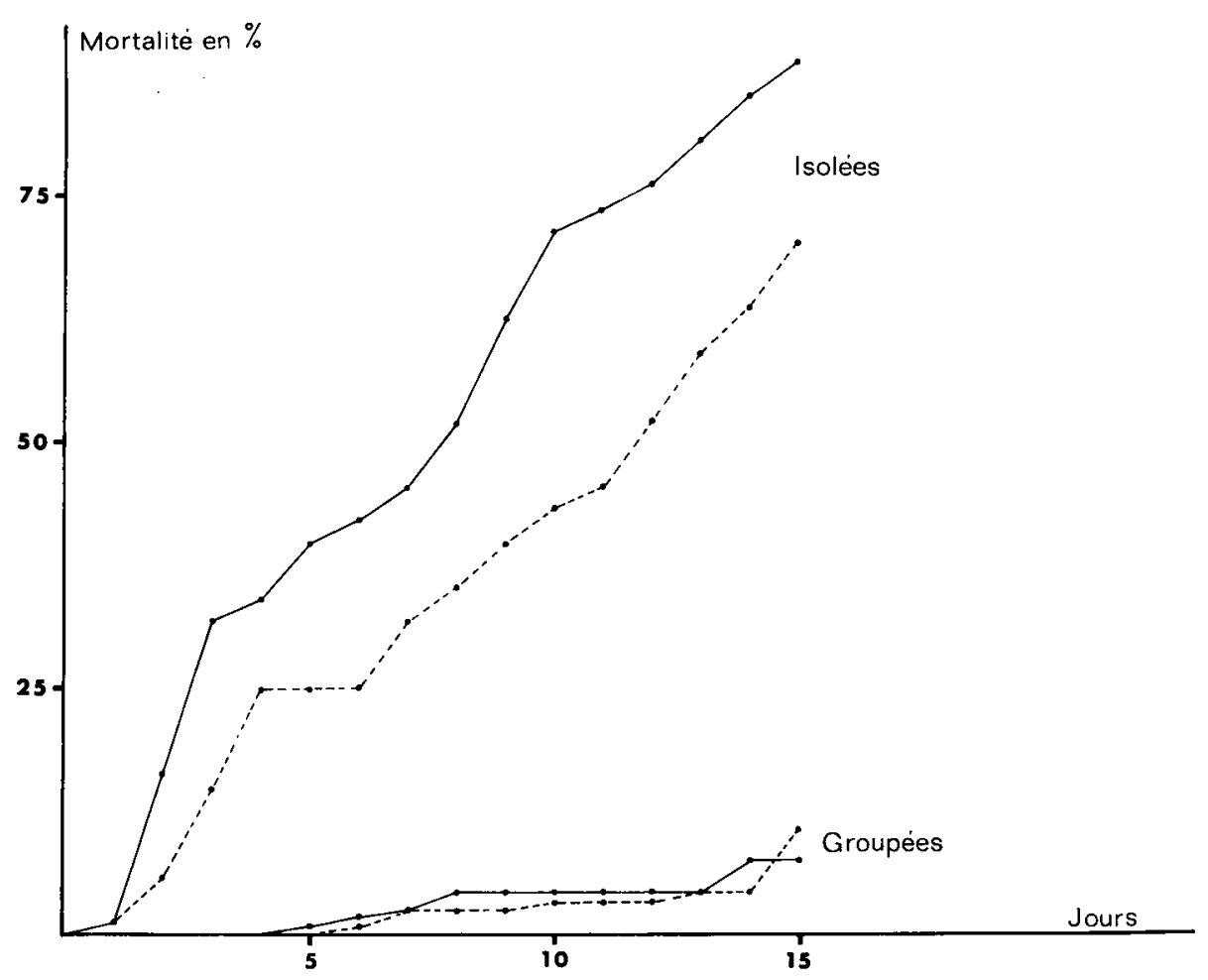

Fig. 5. - Action des extraits lipidiques de gelée royale

: Abeilles traitées disposant de pollen et de candi + extraits lipidiques provenant de $10 \%$ de gelée royale.

Abeilles témoins disposant de pollen et de candi pur.

Авв. 5. - Wirkung der Königinnenfuttersaft-Extrakte

Versuchsbienen, mit Pollen und Futterteig, dem Extrakte aus $10 \%$ Königinnenfuttersaft zugegeben wurden, ernährt.

: Kontrollbienen, denen Pollen und reiner Futterteig geboten wurde.

Les extraits de gelée royale ont donc une action négative sur la survie des abeilles d'été isolées.

Cependant, il est à noter que dans le cas du candi à $20 \%$, la différence de mortalité entre abeilles isolées traitées et témoins n'est significative qu'à $10 \%$. D'autres expériences, qu'il convient d'approfondir, me font penser que cette différence n'est pas imputable à la concentration du candi en extraits de gelée royale, mais en liaison avec la saison où a été prélevé le couvain naissant. 


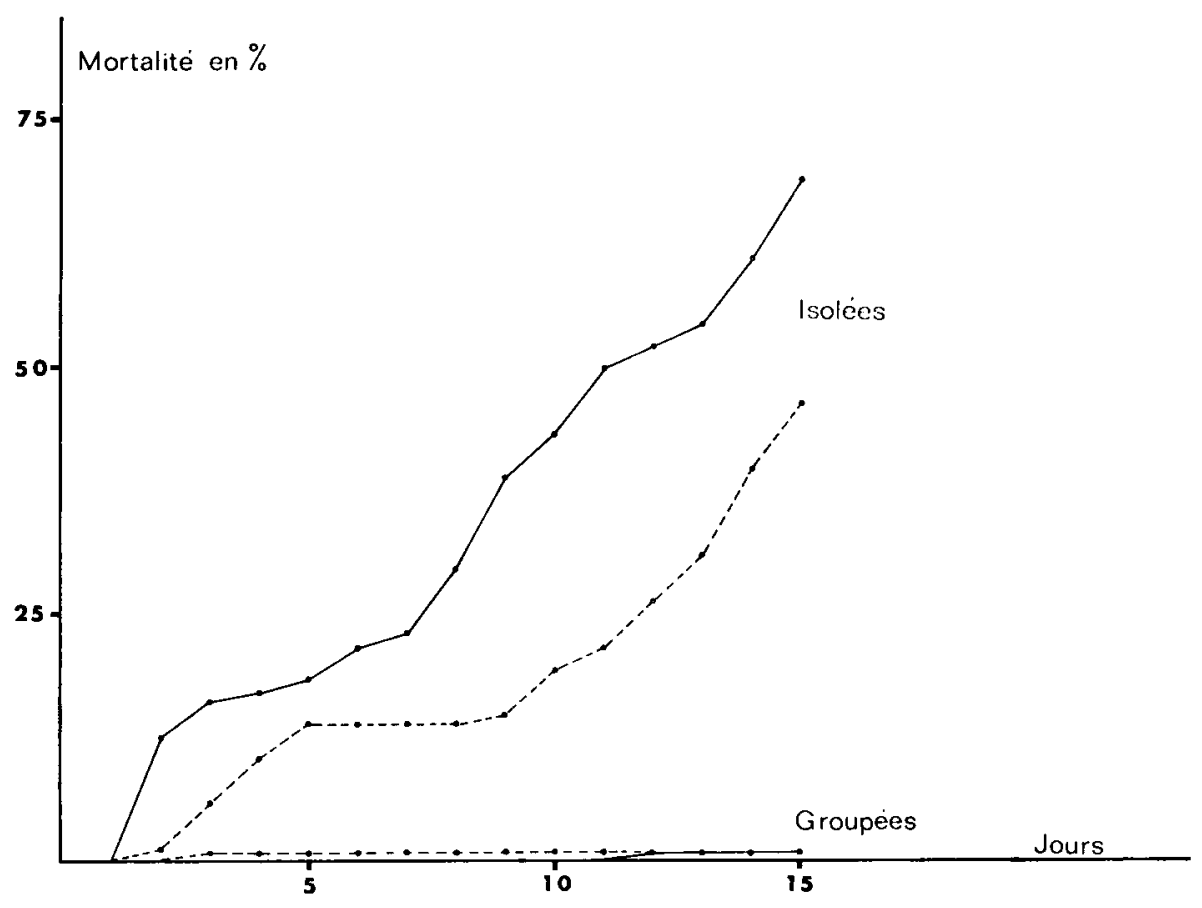

Fic. 6. - Action des extraits lipidiques de gelée royale :- Abeilles traitées disposant de pollen et de candi + extraits lipidiques provenant
de $5 \%$ de gelée royale.

: Abeilles témoins disposant de pollen et de candi pur.

Aвв. 6. - Wirkung der Königinnenfuttersaft-Extrakte

— : Versuchsbienen, denen Pollen und Futterteig mit Extrakten aus 5 \% Königinnenfuttersaft geboten wurde,

: Kontrollbienen, denen Pollen und Futtertejg ohne Zusatz geboten wurde.

Quoiqu'il en soit, ces expériences montrent qu'un régime alimentaire à base d'extraits de gelée royale diminue la survie des abeilles d'été isolées.

\section{DISCUSSION}

\section{A. - Les effets de l'alimentation liquide}

Toutes les expériences relatives à cette question montrent que les abeilles sont sensibles à la nature physique des aliments.

Les abeilles groupées nourries de miel pur ou de miel additionné de gelée 
royale meurent aussi vite que les abeilles isolées suivant le même régime alimentaire, alors que lorsque la nourriture est solide, la différence de mortalité entre abeilles groupées et isolées est toujours hautement significative. Cet effet positif du groupe sur la survie disparaît donc sous l'influence d'une alimentation liquide.

Ces résultats confirment ceux de Chauvin (1952) qui note une mortalité proportionnelle au groupement quand les abeilles disposent de " sirop de sucre à $20 \%$ comme unique nourriture \%. Nous pouvons donc, dès à présent, conclure que ce n'est pas le miel ou le sirop de sucre qui importe, mais le fait qu'il s'agit d'une alimentation liquide.

Au contraire, Maurizio (1946) pense que l'aspect liquide ou solide de la nourriture est sans influence sur la survie quand les abeilles disposent par ailleurs d'eau pure.

Chauvin a expliqué cette divergence de résultats par le fait qu'il n'a pas, comme Maurizio, renouvelé quotidiennement le sirop de sucre et considère comme possible une fermentation.

Je ne partage pas cette hypothèse, et ce pour plusieurs raisons.

La première est que je retrouve les mêmes résultats que lui en dépit du fait que j'ai renouvelé le miel environ tous les deux jours. Par ailleurs, je considère comme peu probable une fermentation du miel à $32^{\circ} \mathrm{C}$ et surtout en un laps de temps aussi bref. Enfin, comme nous le verrons plus loin, la mortalité des abeilles isolées vivant sur miel n'est guère différente de celle des abeilles isolées nourries de candi. Or, s'il y a fermentation du miel chez les groupées, il doit en être de même pour le miel des isolées et ce d'autant plus que, dans ce cas, le renouvellement du miel n'a eu lieu que tous les quatre jours environ.

Pour ma part, je considère donc que les abeilles groupées ont une survie nettement supérieure quand elles sont soumises à un régime alimentaire " solide".

Pour les abeilles isolées, CHAuvin considère qu'elles vivent aussi longtemps sur sirop de sucre que sur candi. Mais personnellement j'émets quelques réserves quant à cette conclusion. Certes, comme je l'ai signalé, il n'y a pas de différence de mortalité significative entre abeilles isolées vivant sur miel et celles vivant sur candi, après dix-sept jours d'isolement, ce qui tend à confirmer les résultats de Chauvin.

Mais, j'ai également noté que si l'expérience avait été poursuivie quelques jours de plus, il en aurait été autrement, puisque déjà après douze jours d'isolement on assiste à une inversion des courbes de mortalité que j'ai attribuée à une accumulation d'eau progressive au cours de la vie claustrée, c'est-à-dire à un effet tardif de l'alimentation liquide.

Par ailleurs, j'ai signalé que si les courbes s'inversent au douzième jour en faveur des isolées vivant sur candi, cela implique qu'avant cette date la mortalité des abeilles isolées disposant de miel est inférieure à celle des isolées vivant sur candi.

Cependant, il n'en demeure pas moins vrai qu'après dix-sept jours d'isolement, il n'y a pas encore de mortalité excessive chez les abeilles isolées vivant sur miel, ce qui exclut à priori toute idée d'une fermentation du miel pendant cette période.

J'en conclus donc que les abeilles isolées supportent mieux que les abeilles groupées les aliments liquides, mais contrairement aux conclusions de Chauvin, 
cela n'est valable que pendant un certain temps, au-delà duquel la rétention d'eau agit tout comme chez les abeilles groupées.

Pendant cette période, l'alimentation liquide a même tendance à améliorer la survie des abeilles isolées par rapport à celle des abeilles isolées vivant sur candi.

En résumé, les abeilles claustrées, isolées ou groupées sont sensibles à l'état physique de la nourriture. On favorise la survie des abeilles groupées par une nourriture solide. En ce qui concerne les abeilles isolées, pour une expérience de courte durée, on a intérêt à utiliser une alimentation liquide, mais si l'expérience prévue est longue il convient d'utiliser là aussi des aliments solides.

\section{B. - Les effets de la gelée royale}

L'action de la gelée royale entière a également été étudiée par Chauvin. L'auteur ne constate aucun effet favorable sur la survie et ce, que la gelée royale soit donnée pure ou mélangée à du miel ou à du sirop de sucre. A mon avis, il n'est pas surprenant que la gelée royale pure distribuée " en frottis contre la toile métallique de la cage " n'ait eu aucune action. En effet, l'expérience préliminaire que j'ai réalisée dans cette optique a révélé que les abeilles claustrées isolées ou groupées n'en consommaient pas.

En ce qui concerne la gelée royale mélangée à une source liquide d'hydrates de carbone, j'ai aussi constaté qu'elle était sans action sur les abeilles groupées. Mais chez les abeilles isolées, si cette action paraît inexistante, il semble que ce ne soit en réalité qu'un artéfact provoqué par l'action simultanée de la rétention d'eau. En effet, quand on dissocie les deux actions en tenant compte du facteur temps d'isolement, on peut constater une action négative de la gelée royale sur la survie.

Ainsi, je considère, comme Chauvin, que la gelée royale n'a aucun effet favorable sur la survie des abeilles claustrées et $j$ 'ajoute que cet effet est au contraire négatif dans le cas des abeilles d'été isolées.

Les travaux relatifs au rôle des extraits lipidiques de la gelée royale se sont multipliés après l'analyse biochimique de cette substance réalisée par BuTENANDT et Rembold (1957-1958). Mais ils n'ont porté que sur des larves d'abeilles (Pain, 1968).

L'intérêt de cette étude chez les abeilles adultes est qu'elle permet de dissocier l'action de la gelée royale de celle de la rétention d'eau. Par ailleurs, elle met en lumière le fait que si dans certains cas, l'action négative de ces extraits est statistiquement significative, dans d'autres, elle est nulle. J'ai alors émis l'hypothèse que cette différence était en relation avec la période où a été prélevé le couvain. Cette idée a été confirmée par une expérience non décrite, portant sur des abeilles d'hiver, où les extraits de gelée royale n'ont eu ni action favorable ni action négative sur la survie des abeilles. Autrement dit, la gelée royale ne serait susceptible de diminuer la survie que chez les abeilles d'été isolées. Il est évident que cette hypothèse mérite d'être vérifiée de façon plus approfondie. 


\section{CONCLUSION}

Les expériences décrites ci-dessus montrent que les abeilles d'été claustrées sont sensibles à l'état physique de la nourriture.

L'action de l'alimentation liquide est immédiate chez les abeilles groupées et plus tardive chez les abeilles isolées.

Au contraire, l'action de la gelée royale entière ou sous forme d'extraits à l'éther ne semble affecter que les abeilles isolées, et ce, de façon plus ou moins accentuée, peut-être en fonction de la période de prélèvement du couvain naissant.

Reçu pour publication en juillet 1970.

Eingegangen im Juli 1970.

\section{ZUSAMMENFASSUNG}

Für die Versuche wurde schlüpfende Sommerbrut verwandt. Die Bienen wurden auf verschiedene Weise ernährt : mit reinem, flüssigem Honig, Futterteig, reinem Königinnenfuttersaft und mit Futtersaft-Extrakten. Alle Bienen waren mit pulverisiertem Pollen versorgt. Wenn den Bienen Futterteig, Pollen oder Königinnenfuttersaft gleichzeitig in drei verschiedenen Futtergeschirren geboten wurde, liess sich eine Abneigung der Bienen gegen den Königinnenfuttersaft feststellen. Deshalb wurde bei diesen Versuchen der Futtersaft mit Honig oder Futterteig vermischt verabreicht.

Die Sterblichkeit ist in den Bienengruppen, die reinen Honig erhielten, sehr hoch. Der Hauptgrund hierfür ist in einer sehr starken Wasserretention zu sehen. Bei den isoliert gehaltenen Bienen zeigen sich die Unzuträglichkeiten der flüssigen Nahrung weniger rasch. Die Sterblichkeitsziffern der Bienengruppen, die mit einer Mischung von Honig und $10 \%$ Königinnenfuttersaft ernährt wurden, waren ebenfalls sehr hoch, was zweifellos auch auf eine Wasserretention zurückzuführen ist. Ein statistisch gesicherter Unterschied in der Sterblichkeit bei einzeln und in Gruppen gehaltenen Bienen liess sich nicht feststellen (Abb. 1). Königinnenfuttersaft scheint die Lebensdauer isoliert gehaltener Bienen zu verkürzen (Abb. 2).

Bei einer Fütterung mit Futterteig scheint die Gruppe von grossem Einfluss auf das Uberleben der Bienen zu sein. Dagegen ist der Unterschied in der Sterblichkeit unbedeutend, wenn die Bienen mit Honig gefüttert werden (Abb. 3). Gekäfigte Bienen scheinen demnach für die physikalischen Eigenschaften der Nahrung empfindlich zu sein.

In anderen Versuchen wurden Extrakte von Königinnenfuttersaft in Futterteig geboten. Es zeigte sich, dass die Königinnenfuttersaft-Extrakte ohne Einfluss auf die Lebensdauer von Bienengruppen sind. Von negativem Einfluss dagegen sind sie auf die Lebensdauer einzeln gehaltener Sommerbienen (Abb. 4-6). Mit anderen Worten : eine Ernährung auf der Grundlage von Königin. nenfuttersaft-Extrakten verkürzt die Lebensdauer isoliert gehaltener Sommerbienen.

\section{REFERENCES BIBLIOGRAPHIQUES}

Butenandt A., Rembold H., 1957. Uber den Weiselzellenfuttersaft der Honigbiene, I. Isolierung, Konstitutionsermittlung und Vorkommen der 10-Hydroxy- $\Delta^{2}$-decensaüre. Hoppe-Seyler's $Z$. physiol. Chem., 308, 284-289. 
Butenandt A., Rembold H., 1958. Uber den Weiselzellenfuttersaft der Honigbiene, II. Isolierung von 2-Amino-4-hydroxy-6(1-2-dihydroxy-propyl)-pteridin. Hoppe-Seyler's $Z$. physiol. Chem., $311,79-83$.

Chauvin R., 1952. Sur le déterminisme de l'effet de groupe chez les abeilles. Physiol. Comp. Ecol., $1,1-7$.

Maurizio A., 1946. Beobachtungen über die Lebensdauer und den Futterverbrauch gefangen gehałtener Bienen. Beih. Schweiz. Bienenztg., 2, (13), 1-44.

PaIN J., 1968. La biochimie des nourritures larvaires des reines et des ouvrières d'abeilles (Apis mellifica L.). Revue des travaux du Max Planck Institut. Ann. Abeille, 11, (1), 49-62.

Sitbon G., $1967 b$. 一 L'effet de groupe chez l'Abeille. I. L'Abeille d'hiver, survie et consommation de candi des abeilles isolées ou groupées. Ann. Abeille, 10, (2), 67-82.

Sirbon G., 1967 c. — L'effet de groupe et la mortalité des abeilles d'hiver et d'été, isolées et groupées. Ann. Abeille, 10, (4), 203-212.

Sitbon G., 1968 a. - Les sucres totaux chez l'Abeille d'hiver en fonction de l'isolement. Insectes sociaux, 15, (1), 37-44.

Sitbon G., 1968 b. - Teneur en eau et en azote chez l'Abeille en fonction de l'isolement. Insectes sociaux, 15, (4), 413-418. 